\title{
Evaluating the 3D Structures of PtNi Nanoparticles from Single Projection ADF STEM Images and EDX Maps
}

\author{
Katherine E. MacArthur ${ }^{1}$, Marc Heggen ${ }^{1}$ and Rafal E. Dunin-Borkowski ${ }^{1}$ \\ 1. Ernst Ruska-Centre for Microscopy and Spectroscopy with Electrons and Peter Grünberg Institute \\ Forschungszentrum Jülich, Germany.
}

Pt-based bimetallic nanoparticles have demonstrated great promise as catalysts for the oxygen reduction reaction (ORR) in hydrogen fuel cells [1]. The addition of a second transition metal, such as Ni or Co, not only reduces the cost of the catalyst due to the reduction in Pt metal loading, but also provides an increase in activity. Recently, octahedral PtNi nanoparticles have attracted considerable interest due to the exceptionally high activity for the ORR demonstrated by $\mathrm{Pt}_{3} \mathrm{Ni}$ (111) [2]. A strong link has been demonstrated between the atomic-scale structures of octahedral nanoparticles and their catalytic performance in a number of studies. Advances in catalysis rely on the synthesis and characterisation of nanoparticles that have carefully tailored structures and compositions. Therefore, high-resolution threedimensional (3D) characterisation is an essential step in their further improvement.

Transmission electron microscopy (TEM) is arguably the only technique, which provides particle-specific information about the structure and composition of individual nanoparticles. A combination of annular dark-field scanning TEM (ADF-STEM) and either energy dispersive X-ray (EDX) analysis or electron energy-loss spectroscopy (EELS) provides essential sub-nanometre compositional information on a particle-by-particle basis. Unfortunately, TEM suffers from a well-known problem, that it produces twodimensional (2D) images of 3D structures. Therefore, a great deal of uncertainty can still exist about the particle structures due to insufficient information in the third dimension. Such a problem persists for most signals captured in TEMs, including ADF-STEM, EDX and EELS. Although EDX tomography in principle provides the 3D information required, many nanoparticle structures are unable to survive the high electron dose they would be subjected to during the process. Furthermore, the more information that can be understood from single orientation EDX maps, the greater the possibility of following morphological changes during in situ experiments under gas environments.

ADF STEM images and EDX maps were simulated for a selection of possible PtNi octahedron structures at a variety of sample tilts, examples can be seen in Figure 1 showing the Pt hexapod and the Pt hexapod and edges structures. The focus of this study is on understanding how 2D maps can be related back to the $3 \mathrm{D}$ structures that produced them. The compositions of the models were kept as close to 40 at $\% \mathrm{Pt}$ as possible, so that subtle details in the STEM images and EDX maps could be compared without needing to consider any variations in average composition. An important factor that was investigated was whether the measurement of shell thickness is affected by sample tilt (see Figure 2). The width of the Pt peaks at the edges of the nanoparticle increases with sample tilt angle because the columns of atoms causing this peak become less parallel to the electron beam direction. However, the distance between where the signals fall to zero remains approximately constant with sample tilt angle (black arrows in Figure 2) and the measured shell thickness varies by less than $0.1 \mathrm{~nm}$. Differentiation between many of the structures relies on subtle variations in intensity within the particle and whether or not these variations are above the noise level for a particular pixel dwell time. Such a library of simulations and its analysis is essential for optimising time at the electron microscope, which can often be limited [3][4]. 


\section{References:}

[1] O.T. Holten, J. W. Stevenson Platin. Met. Rev. 57 (2013) p. 259-271.

[2] V.R. Stamenkovic et al. Science 3155811 (2007) p. 493-497.

[3] K.E. MacArthur, M. Heggen, R.E. Dunin-Borkowski. Adv. Struct. Chem. Imaging Accepted

[4] K. M. acknowledges the Helmholtz Association for funding this research through their Postdoctoral Research Fellowship. M. H. is grateful to the Deutsche Forschungsgemeinschaft (DFG) for support through grant HE 7192/1-1. M. H. and R. D.-B. are grateful to the European Union for support through H2020-NMP-23-2015 grant (project number 686053). Martin Gocyla is thanked for useful discussions.
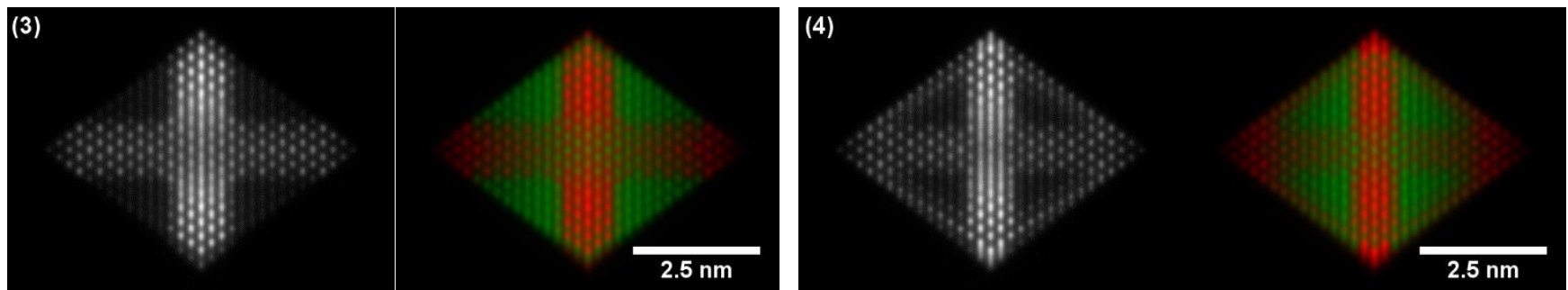

Figure 1. Two example simulated structures showing both the ADF STEM image and the EDX map close to a $<110>$ orientation for Pt hexapod (3) and Pt hexapod and edges (4). The Pt-L line is shown in red, the $\mathrm{Ni}-\mathrm{K}$ is in green.

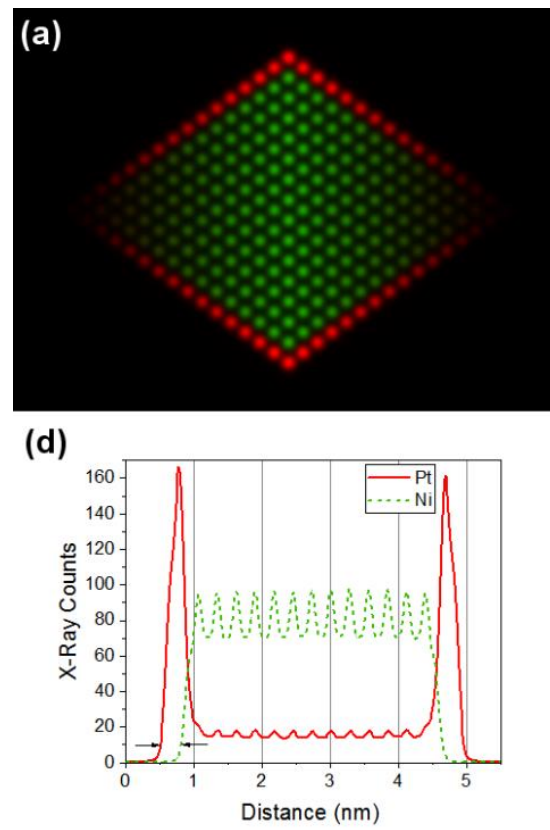

\section{(b)}

(c)

$2.5 \mathrm{~nm}$

(e)

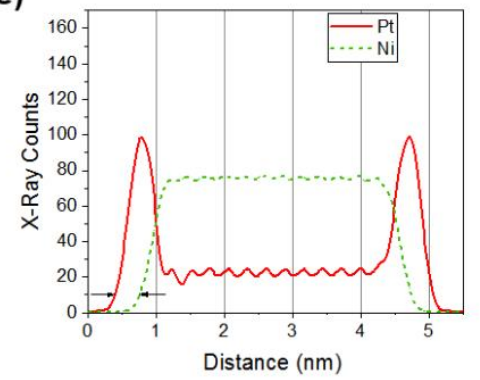

(f)

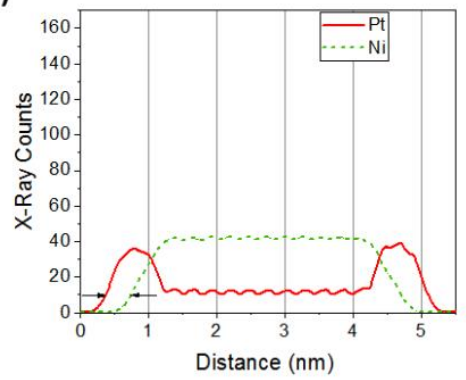

Figure 2. Simulated EDX maps of nanoparticles comprising a Pt monolayer shell with a Ni core viewed (a) along $\langle 110\rangle$, (b) tilted by $5^{\circ}$ from $\langle 110\rangle$ and (c) tilted by $10^{\circ}$ from $\langle 110\rangle$. The intensity in (b) and (c) has been doubled to make the figures visible on the same intensity scale as in (a). Corresponding line profiles taken vertically through the centre of the nanoparticle are shown in (d), (e) and (f), assuming a dwell time of $1 \mathrm{~s}$ per point and a pixel width of $15 \mathrm{pm}$. The Pt-L signal is shown in red, while the Ni-K signal is shown in green. The approach used to measure the shell thickness is marked using arrows. 